\begin{tabular}{c} 
International Journal of Engineering \& Technology, $7(2.14)(2018) 110-113$ \\
International Journal of Engineering \& Technology \\
SPC \\
Website: www.sciencepubco.com/index.php/IJET \\
Research Paper \\
\hline
\end{tabular}

\title{
Onmyouji: Gesture-based virtual reality game
}

\begin{tabular}{|c|}
\hline Ka Weng Chou, Albert Quek*, Hui Hong Yew \\
\hline Multimedia University, Malaysia \\
\hline *Corresponding author E-mail: quek.albert@mmu.edu.my \\
\hline
\end{tabular}

\begin{abstract}
With the emergence of Virtual Reality (VR), user's senses are taken into consideration in achieving a natural and intuitive humancomputer interaction (HCI) as well for a more effective data interaction. The conventional keyboard and mouse interface and control methods of VR no longer sufficiently handles the richness of the information and facilitate intuitive user interaction in the virtual environment. There are alternative controllers such as motion controller used in the game, but this burden users for having them to hold a controller during gameplay. Hand gestures are a powerful tool for communication among humans. Hand gestures recognition has the potential to achieve the naturalness and intuitiveness for HCI. Here, we have proposed a VR game implemented a set of simple gestures with Leap Motion controller named Onmyouji. Onmyouji emphasizes on the VR experience provided with utilizing hand gestures to move and cast attack as the player control. A set of gestures are designed to fulfill the naturalness and intuitiveness of controls in the game. By qualitative user evaluation on a target interest group, most of the participants evaluated positively on the intuitive gestures and gameplay. For the overall game experience, all participants rated 4 and 5 out of 5 scores.
\end{abstract}

Keywords: Gesture-based game; Gesture mechanics; Intuitive gesture; Leap motion; Virtual Reality;

\section{Introduction}

Since 1990s, rapid development in computer technologies have made a flood of demand for more efficient user-computer interaction. This new demand has prompted significant interest in research of more natural communication modalities such as speech, gestures and eye gaze. Thus, multimodal interface serves as an abstract layer with the ability to process inputs in multiple modalities which a computer is capable of extracting useful information and translate it into program commands [1]. To achieve a more natural HCI, sense and gestures of users can be used. On the other hand, Virtual Reality (VR) is defined as a virtual environment generated by using computer in which the users have a sense of being in it [2] which it facilitates and involves human senses for more intuitive data interactions [3].

In [4] stated that VR provides high immersion, interaction and dynamic multidimensional digital information to the user and by incorporating Leap Motion, gesture control makes natural HumanComputer Interaction (HCI) possible to be achieved. The use of Kinect for gesture detection is also commonly used in game research and it provides a certain degree of immersion of gameplay to the player. For example, a group of Multimedia University researchers have developed a gesture-based First-Person Shooter (FPS) game called The Invoker that utilizes hand and body movements to perform physical action such as dodging and spell casting [5]. In Zhejiang University, the researchers have presented a double hand-gesture interaction (DHGI) method for walkthrough in VR using Oculus Rift and Leap Motion. According to the user feedback, with the use of DHGI, it significantly improves immersion and reduces the sense of motion sickness in VR [6]. With the use of gesture-based input, conventional keyboard and mouse interface are no longer sufficient to handle the richness of the information exchange in $\mathrm{HCI}$ [7].
There are also systems such as PlayStation Move and Nintendo Wiimote that use motion control as input for games, but the user is required to hold physical devices or controllers during gameplay [8]. In a paper where 3 controllers were compare using Fitts' law task as per ISO 9241-9, the Nintendo Wii Remote for infrared pointing, the Nintendo Classic Controller for analogue stick pointing and a standard mouse as a baseline condition. Result shows that 14 out of 15 participants preferred the Wiimote over Classic Controller for the use in interactive home entertainment environment [9]. Using Wiimote as motion controller for VR game might not necessary be the best option as it imposes a degree of inaccuracy where the controller requires to be pointed in a certain direction, for a more accurate sensing. Thus, a unique configuration is needed for the approach [10]. Researchers have tested on a configuration that uses 2 gestured-based input devices such as Kinect 2 and Leap Motion to support full-body interactions in the form of hand and body gestures. As the result, players felt more natural in performing gestures and body movements as compare to handheld motion controllers [11].

For that, this research is to enhance HCI and immersion in VR by allowing player to physically use hand gestures as a game input to control and manipulate objects in the game without having the player to hold any physical devices or controllers during gameplay.

In this paper, we propose a gesture-based VR game called "Onmyouji" that takes hand gestures as player control with the use of a Leap Motion controller and a smartphone gyroscope and accelerometer. With the set of simple and intuitive hand gestures, player can move and cast attack in the game world. This provides a more natural user interactions and facilitates an immersive VR experience for the user. Visual and audio feedback are adopted in game to heighten user experience. The rest of the paper are organized as follows. Section 2 introduces the game concept of Onmyouji, user interface and level. Section 3 describes the set of 
gestures and its implementation in the proposed system. Finally, section 4 presents the result of user evaluation on the proposed system while section 5 concludes the paper.

\section{Game design}

\subsection{Game concept}

Onmyouji is a gesture-based virtual reality (VR) game, which emphasizes on hand gestures in movement control and attack Meaning that the player is able to perform in-game actions such as moving character or attacking enemies by performing certain hand gestures. The game comes with simple gameplay and interfaces to provide immersive experience to the target audience with the use of VR. No physical controllers are needed for the game. Player is only required to wear a head-mounted display (HMD) and he/ she can roam in the virtual environment and play the game with bare hands. The player will be given objective to defeat enemies and level boss in the game. Figure 6 shows a summary of the gestures and gameplay of the game.

\subsection{User interface}

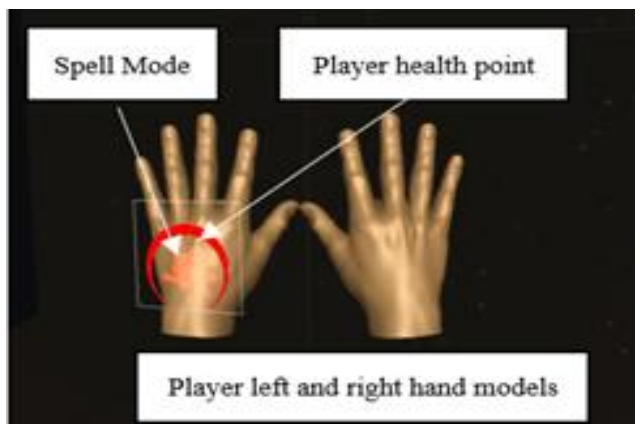

Fig. 1: In-game hand model

Figure 1 refers to the in-game visual where player get to see his/her hands appear out in a virtual form. Basically by looking at both of their hands, player's health bar will appear on the left hand. The Spell Mode will also appear on the player's left hand informing the player on the current spell (fire, water, earth or lightning). By looking at the player's left palm the start menu and tutorial will appear (Figure 2). This is to assist the player to navigate to different levels.

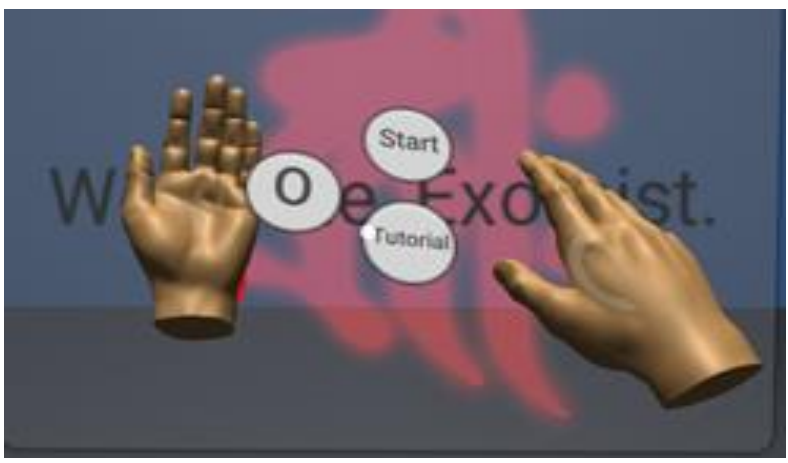

Fig. 2: In-game hand model

\subsection{Game Level}

Onmyouji consist of a tutorial and a game level. In the tutorial, player will learn all the gestures which include movements and spell casting. Whereas the game level, player moves from the starting point to the end of the level. As player moves forward player will have to eliminate any enemies on sight. In the end of the level, player will encounter a final boss (the last enemy). Defeating the boss to complete the level (Figure 8).

\section{Game implementations}

\subsection{Hardware and software}

The hardware and software being adopted and implemented in this project are as below:

- Head-mounted display (VR Box)

- Leap Motion controller

- Android smartphone (Xiaomi Redmi Note)

- A laptop

- TrinusVR (Software and mobile application)

- Unity3D game engine

The game is developed using Unity3D game engine and run on a laptop. The game screen is mirrored to the Android smartphone's screen using TrinusVR [12], a commonly used software and mobile application that allows the casting of in-game visuals to smartphone screen. It utilizes the smartphone's built-in accelerometer and gyroscope to control the game view according to the user's head orientation to facilities VR. In order to have the game VR-enabled, the smartphone is place into the VR Box, a VR headmounted display (HMD). The Leap Motion [13] controller is set in front of the VR Box to detect and recognize gestures during the game (Figure 3).

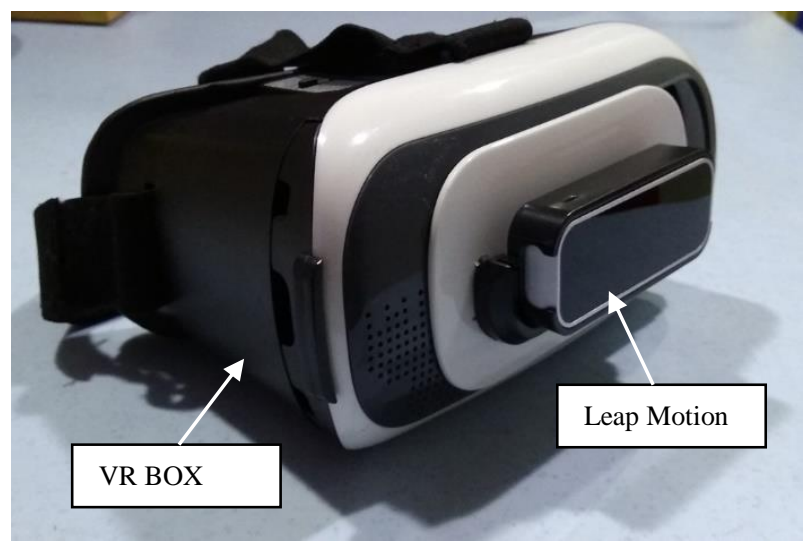

Fig. 3: Simple configuration of Leap Motion and VR Box

\subsection{Gestures}

The core mechanics of gesture control in the game comprise of the following:

- Movement Control - To move player forward and backward.

- Hand Ray Control - To trap enemies in a cube.

- Spell Control - To choose spells such as fire, water, earth or lightning.

- $\quad$ Attack Control - To create energy balls and attack enemies.

\subsubsection{Movement control}

Movement control allows player to move forward and backward in the game. The player can only use the movement control if left hand is open and not pinching or in a "holding fist" gesture (Figure 4). An input simulator is used to simulate key presses such as key "W" and " $\mathrm{S}$ " in moving the character in the game. The hand palm's position is the distance of the player's hands relative to the Leap Motion controller. In the following, the pseudocode for movement control is stated. 
IF Left Hand is pushed forward THEN

Press button "W"

Move forward

ELSE

Release button "W"

ENDIF

IF Left Hand is pulled backward THEN

Press button "S"

Move backward

ELSE

Release button " $S$ "

ENDIF

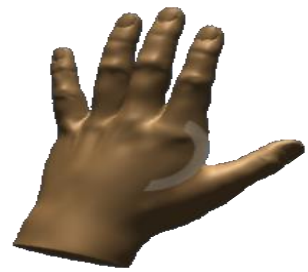

Fig. 4: Left hand gesture for movement control

\subsubsection{Hand ray control}

Hand ray control allows player to trap enemies in a cube and it can only be activated if left hand is in a "grabbing fist" gesture. Whenever the left hand is in "grabbing fist" gesture, an arrow will be casted from right hand palm towards a certain range and ray casting is done to detect collision with enemies. A tile will be casted below an enemy if the arrow collides with an enemy (Figure 5). If an enemy is "highlighted", the grabbing right hand fist will cast a cube to trap the enemy. In the following, the pseudocode for hand ray control is stated.

1 IF Left Hand is grabbing THEN

2 Cast ray from player's right hand to a certain range

3 IF currentTarget is not previousTarget THEN

$4 \quad$ Remove tile below the enemy

$5 \quad$ ELSE

6 Cast tile below the enemy

7 ENDIF

8 ENDIF

9 IF Right Hand is grabbing THEN

10 Cast cube to trap the enemy

11 ENDIF
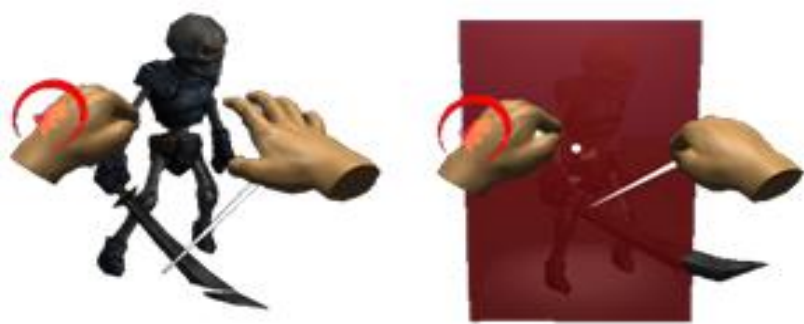

Fig. 5: Casting arrow and trapping enemy

\subsubsection{Spell control}

Spell control allows player to hand-drawn shapes to trigger different element-switching which are circle for "Fire", square for "Water", triangle for "Earth" and star for "Lightning" (Figure 6). Aside left hand for movement control, player is required to use right hand with index finger pointed out only to trigger the game in recognizing shape-drawings. If player has successfully drawn a shape, current element will switch to the respective element represented by the shape, else nothing happens.

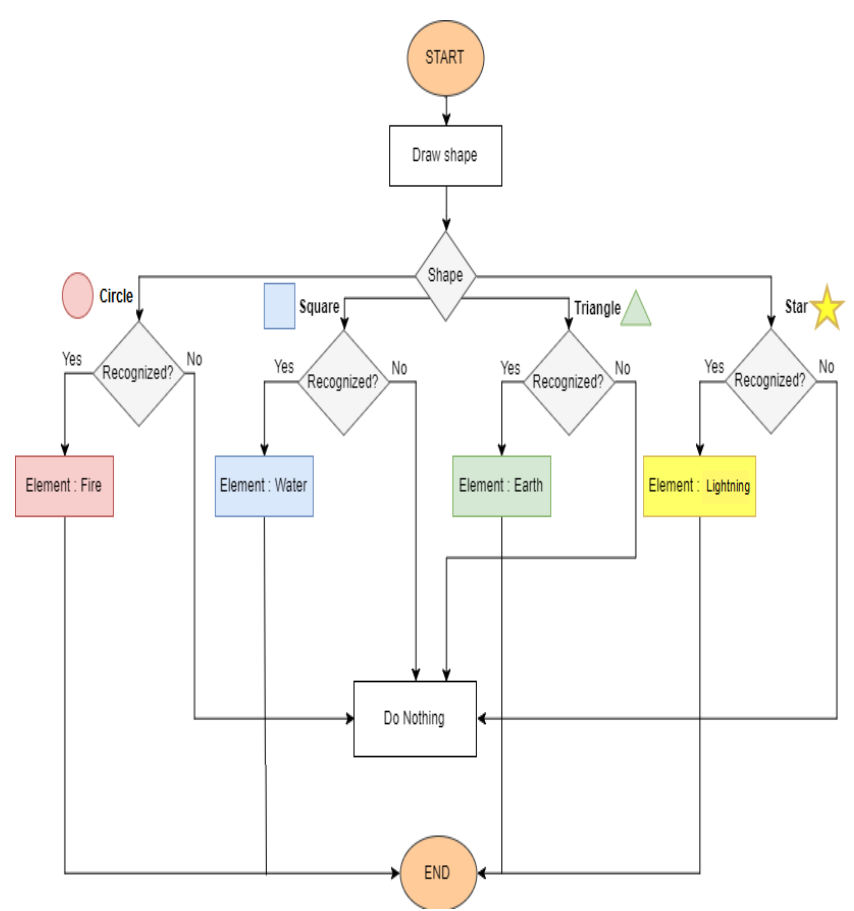

Fig. 6: Flowchart for spell control

\subsubsection{Attack control}

Attack control allows player to create energy balls that can cause damages to enemies. It can only be activated if the left and right hand are pinching and pull away each other to a certain distance (Figure 7). In the following, the pseudocode for attack control is stated.
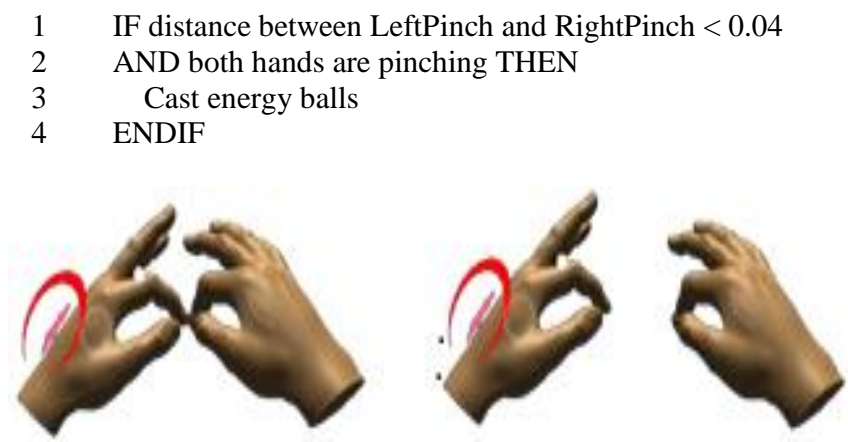

Fig. 7: Pinching and pulling to perform attack

\section{User evaluations}

Onmyouji was created to introduce gesture-based mechanics into a Leap Motion-based VR game. Hence, the target audience were casual gamers who have interest in VR games. The game was tested on a group of 14 participants ranging from 18 to 22 years old. All participants were given 15 minutes to try out the game with an option to extend the time frame. Questionnaires had been given to the testers to evaluate the game based on its controls, features, appearances and performances. Based on the questionnaires collected, the responses of the participants are as the following:

- 12 out of 14 testers are willing or possibly willing to purchase the game if it was published with better visual quality and more game features.

- $\quad 85.7 \%$ (rated 4 and 5 scores) of testers are willing to play the game again as they are interested in the gesture-based mechanics. 
- $78.6 \%$ (rated 4 and 5 scores) of testers responded that the virtual hands gave them sense of reality during the game.

- More than $60 \%$ of testers indicated that the gestures are intuitive and meaningful compare to conventional controller.
In summary, the testers were asked to evaluate the overall experience in a scale of 1 to 5,1 is very bad and 5 is very good to wrap up the questionnaire. All the testers rated 4 and 5 scores. This means that testers like the overall experience of the game.

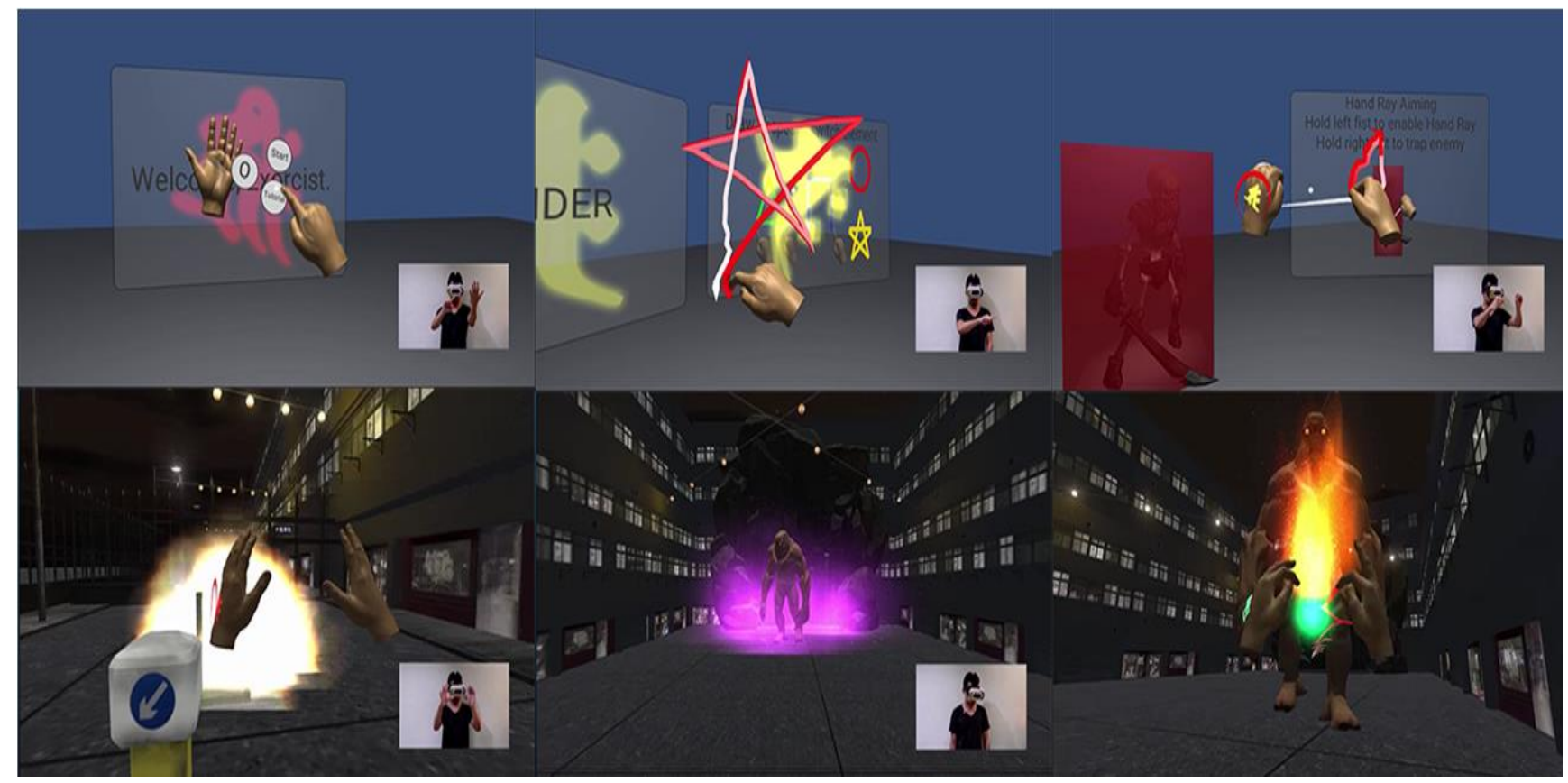

Fig. 8: Screenshots from Onmyouji game depicting various gestures and gameplay. All gestures are captured by Leap Motion sensor. Top row from left: Switching to tutorial level; Spell control to trigger lightning by drawing a star; Grabbing gesture to capture enemy; Moving towards the explosion; Boss appearance in the level; Casting fire to the enemy boss.

\section{Conclusion}

In conclusion, Onmyouji is a gesture-based VR game that uses hand gestures as input with the use of a Leap Motion controller and a smartphone gyroscope and accelerometer for player's head orientation tracking in VR. This user interaction in the form of hand gestures for game input presents a different game control experience apart from conventional game controllers. In the future, we look forward to extend this project by introducing different types of hand gestures as commands and enable more extensive user interaction in the form of in-game virtual hands with the virtual environment.

\section{References}

[1] Bainbridge WS (2004), Berkshire encyclopedia of human-computer interaction. Berkshire Publishing Group LLC.

[2] Jacobson L (1993), On the cutting edge of technology. Sams Publishing.

[3] McLellan H (2004), Handbook of research on educational communications and technology. Lawrence Erlbaum Associates.

[4] Qiu YH \& Luo XJ (2013), Application of computer virtual reality technology in modern sports. Proceedings of the IEEE 3rd International Conference on Intelligent System Design and Engineering Applications, pp. 362-364
[5] Quek A \& See J (2015), The invoker: Intuitive gesture mechanics for motion-based shooter RPG. Proceedings of the Game Physics and Mechanics International Conference, pp. 6-10.

[6] Zhang F, Chu S, Pan R, Ji N \& Xi L (2017), Double hand-gesture interaction for walk-through in VR environment. Proceedings of the 16th International Conference in Computer and Information Science, pp. 539-544.

[7] Murthy GR \& Jadon RS (2009), A review of vision based hand gestures recognition. International Journal of Information Technology and Knowledge Management 2, 405-410.

[8] Boas YA (2013), Overview of virtual reality technologies. Proceedings of the Interactive Multimedia Conference 2013, pp. 1-6.

[9] Natapov D, Castellucci SJ \& MacKenzie IS (2009), ISO 9241-9 evaluation of video game controllers. Proceedings of the Graphics Interface, pp. 223-230.

[10] Chow YW (2008), The Wii Remote as an input device for 3D interaction in immersive head-mounted display virtual reality. Proceedings of the IADIS Multi Conference on Computer Science and Information Systems, pp. 8-92.

[11] Amir MH, Quek A, Sulaiman NR \& See J (2016), Duke: Enhancing virtual reality based FPS game with full-body interactions. Proceedings of the 13th International Conference on Advances in Computer Entertainment Technology, pp. 1-6.

[12] trinusvirtualreality.com (2018), TrinusVR. https://www.trinusvirtualreality.com/.

[13] Leap Motion Inc. (2017), Leap Motion. https://www.leapmotion.com/ . 